\title{
Redox control of glutamine utilization in cancer
}

\author{
L Alberghina ${ }^{\star, 1,2}$ and D Gaglio ${ }^{1,3}$
}

Glutamine utilization promotes enhanced growth of cancer cells. We propose a new concept map of cancer metabolism in which mitochondrial NADH and NADPH, in the presence of a dysfunctional electron transfer chain, promote reductive carboxylation from glutamine. We also discuss why nicotinamide nucleotide transhydrogenase (NNT) is required in vivo for glutamine utilization by reductive carboxylation. Moreover, NADPH, generated by both the pentose phosphate pathway and the cancer-specific serine glycolytic diversion, appears to sustain glutamine utilization for amino-acid synthesis, lipid synthesis, and for ROS quenching. The fact that the supply of $\mathrm{NAD}^{+}$precursors reduces tumor aggressiveness suggests experimental approaches to clarify the role of the NADH-driven redox network in cancer.

Cell Death and Disease (2014) 5, e1561; doi:10.1038/cddis.2014.513; published online 4 December 2014

\section{Facts}

- Sustained growth and survival of most cancer cells rely upon a metabolic rewiring, characterized by an enhanced glycolytic flux and a stimulated utilization of glutamine by reductive carboxylation.

- Mitochondrial dysfunction (for instance Complex I reduced activity) facilitates insurgence of cancer metabolic rewiring.

- $\mathrm{NAD}^{+} / \mathrm{NADH}$ and $\mathrm{NADP}^{+} / \mathrm{NADPH}$ are involved in several reactions of the cancer metabolic rewiring pathways.

- Manipulation of $\operatorname{NAD}(\mathrm{H})$ metabolism in cancer cells affects their tumor forming ability in xenografts.

\section{Open Questions}

- Which is, in various cancer cell types characterized by differential aggressiveness, the quantitative flux of NADPH (coming either from the pentose phosphate pathway or from the serine synthesis, one-carbon-folate- metabolism and glycine cleavage pathway) that ends up in NEAA, in GSH, and in lipids?

- Which is the mitochondrial/cytosolic compartmentalization of NADH and of NADPH metabolism in cancer metabolic rewiring?
- Does the cytoplasmic malate dehydrogenase/malic enzyme pathway convert NADH to NADPH in cancer cells?

Two metabolic adaptations have been recognized as hallmarks of cancer cells: (1) an increased utilization of glucose to produce lactate through the glycolytic pathway ${ }^{1}$ and (2) an enhanced consumption of glutamine by reductive carboxylation to sustain anabolic processes. ${ }^{2}$ Although the metabolic profile of cancer cells may be affected by the tissue that is transformed and by the responsible oncogene ${ }^{3}$ a large number of different cancer types present the indicated metabolic rewiring. It follows that a glucose analog, 2-fluorodeoxy-glucose (FDG), is currently employed in positron emission tomography (PET) for diagnostic purposes. ${ }^{4}$

Several oncogenic signaling pathways, often dependent upon the activation of oncogenes such as ras and myc, found mutated in several human cancers, ${ }^{5-7}$ express their transforming activity by inducing mitochondrial dysfunction (which often causes a reduction of Complex I activity) and by promoting glycolysis and glutamine utilization. ${ }^{6,8-17}$

Inhibition of the OXPHOS mitochondrial pathway, and more specifically of Complex I, is able to promote glutamine utilization. ${ }^{18}$ Both pathways of cancer metabolic remodeling are required to support cancer cell proliferation. In fact both inhibition of mitochondrial glutaminase (GLS2), which catalyzes the hydrolysis of glutamine to glutamate, and inhibition of lactate dehydrogenase $\mathrm{A}$, the enzyme that converts pyruvate

\footnotetext{
${ }^{1}$ SYSBIO Center for Systems Biology, Department of Biotechnology and Biosciences, University of Milano-Bicocca, Milan and Rome, Italy; ${ }^{2}$ Department of Biotechnology and Biosciences, University of Milano-Bicocca, Piazza della Scienza 2, Milan, Italy and ${ }^{3}$ Institute of Molecular Bioimaging and Physiology (IBFM), National Research Council (CNR), Via F.lli Cervi 93, Segrate, Milan, Italy

${ }^{*}$ Corresponding author: L Alberghina, SYSBIO Center for Systems Biology, Department of Biotechnology and Biosciences, University of Milano-Bicocca, Piazza della Scienza 2, Milan, Rome 20126, Italy. Tel: +39 264483515; Fax: +39 264483519; E-mail: lilia.alberghina@ unimib.it

Abbreviations: 1,3BPGA, bisphosphoglycerate; 3PGA, 3phosphoglycerate; 6PGL, 6-phosphogluconolactone; AcCoA, acetyl-CoA; ACL, ATP citrate lyase; Akg, $\alpha$-ketoglutarate; Ala, alanine; Arg, arginine; Asn, asparagines; Asp, aspartate; $\mathrm{CH}^{+}$-Thf, 5-methylenetetrahydrofolate; $\mathrm{CH}_{2}$-Thf, 5, 10-methylenetetrahydrofolate; Cit, citrate; F1,6BP, fructose 1,6 biphosphate; FFA, fatty acids; Fum, fumarate; G6P, glucose 6-phosphate; GA3P, glyceraldehyde 3-phosphate; GDH, glutamate dehydrogenase; Glc, glucose; Gln, glutamine; Glu, glutamate; Gly, glycine; GOT, glutamic-oxaloacetic transaminase; GSH, glutathione reduced; GSSG, glutathione oxidized; Isocit, isocitrate; Lac, lactate; LDHA, lactate dehydrogenase; Mal, malate; MDH 1 and 2, malate dehydrogenase; ME, malic enzyme; MTHFD1 and 2, methylenetetrahydrofolate dehydrogenase (NADP+ dependent); NEAA, non-essential amino acids; NNT, nicotinamide nucleotide transhydrogenase; Oaa, oxalacetate; PDH, pyruvate dehydrogenase; PDHKs, pyruvate dehydrogenase kinases; PPP, pentose phosphate pathway; P-Pyr, 3-phosphohydroxypyruvate; Pyr, pyruvate; R5P, ribose 5-phosphate; ROS, reactive oxygen species; Ser, serine; SHMT1 and 2, serine hydroxymethyltransferase; Succ, succinate

Received 29.8.14; revised 13.10.14; accepted 21.10.14; Edited by G Melino
} 


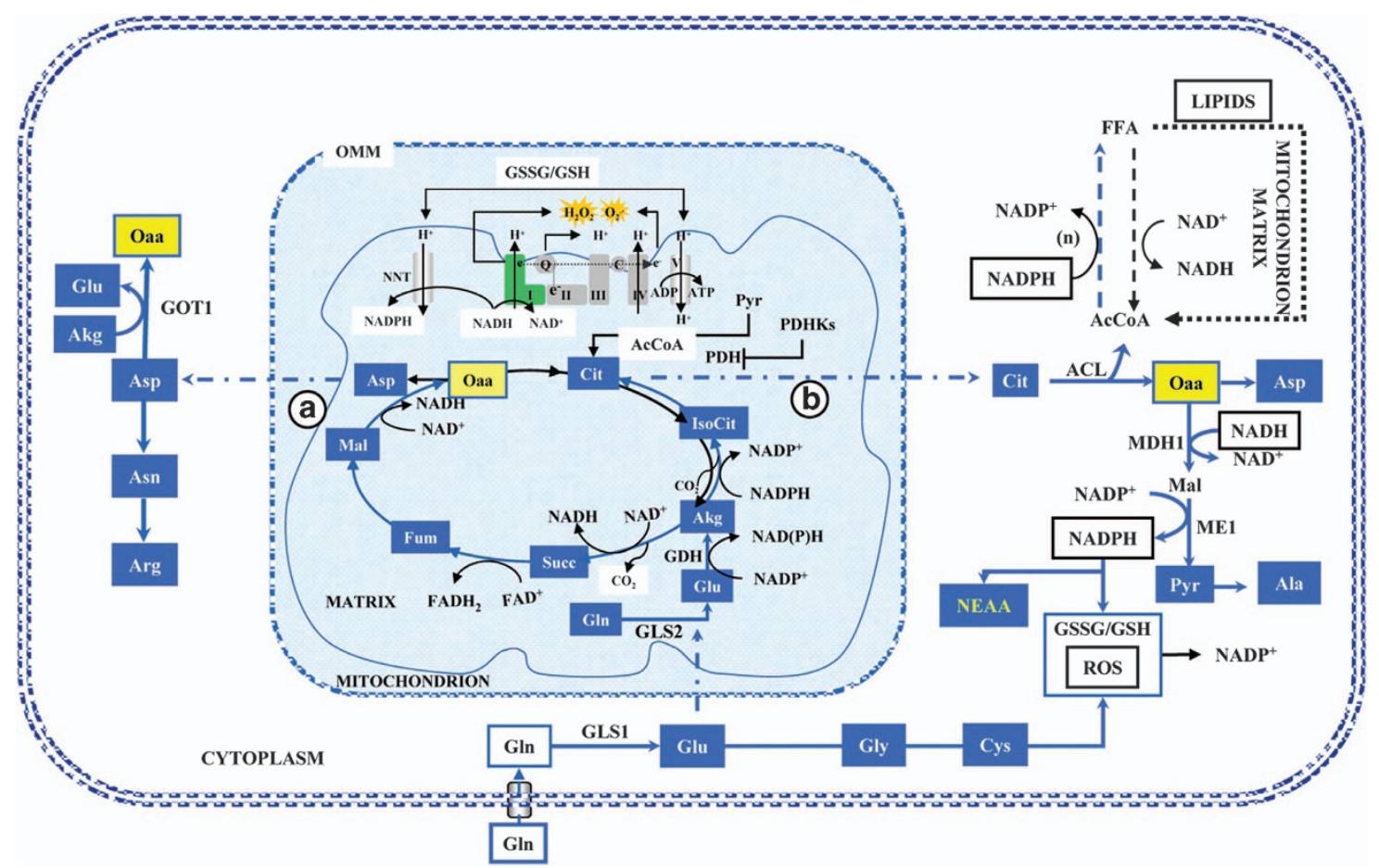

Figure 1 Schematic representation of glutamine metabolic rewiring. Glutamine imported via Slc1a5 enters in a complex metabolic pathway, described in the text, so that both its carbon and nitrogen are utilized to promote growth and survival of cancer cells. AcCoA, acetyl-CoA; ACL, ATP citrate lyase; Akg, $\alpha$-ketoglutarate; Ala, alanine; Arg, arginine; Asn, asparagine; Asp, aspartate; Cit, citrate; Cys, cysteine; FFA, fatty acids; Fum, Fumarate; GDH, glutamate dehydrogenase; Gln, glutamine; Glu, glutamate; Gly, glycine; GOT, glutamic-oxaloacetic transaminase; GSH, glutathione reduced; GSSG, glutathione oxidized; Isocit, isocitrate; Mal, malate; MDH 1 and 2, malate dehydrogenase; ME, malic enzyme; NEAA, non-essential amino acids; NNT, nicotinamide nucleotide transhydrogenase; Oaa, oxalacetate; PDH, pyruvate dehydrogenase; PDHKs, pyruvate dehydrogenase kinases; Pyr, pyruvate; ROS, reactive species oxygen; Succ, succinate

into lactate, are able to suppress tumor cell proliferation. ${ }^{19,20}$ No information is presently available on links between glycolysis and glutamine utilization by reductive carboxylation, although it has been reported that, with few exceptions, ${ }^{21,22}$ cancer cell growth cannot proceed in the presence of only glutamine, with no glucose present. ${ }^{23}$ Taken together, these findings have suggested that metabolic differences of various cancer cells types may open new ways of target identification towards more effective anti-cancer drugs. ${ }^{24,25}$

To reach this aim, it is of course necessary to clarify in detail the cancer-specific metabolic pathways. In this paper, we present a reconstruction of the glutamine and glucose utilization pathways in cancer cells which, in a novel manner, clarifies their regulatory connections with red/ox processes centered on $\mathrm{NAD}^{+} / \mathrm{NADH}$ and $\mathrm{NADP}^{+} / \mathrm{NADPH}$. No coverage is given here on the manifold and complex relationship between pathways of $\mathrm{NAD}(\mathrm{P})^{+}$utilization and on their impact on glutamine and glucose metabolism. The $\operatorname{NAD}(P)^{+}$-utilizing systems include (i) mono ADP-ribosylation reactions, (ii) Poly ADPR polymerases (PARPs), (iii) ADP ribosyl cyclases, and (iv) sirtuins.

\section{Pathways of Glutamine Utilization in Cancer Cells}

The network of biochemical reactions of mammalian cell metabolism is well known and genome-wide reconstructions of human metabolism, specific for many cell types, are available. $^{26}$ Recently, the role of signaling in controlling metabolism by affecting enzyme activities either at the transcriptional or at the translational and the posttranslational levels has been elucidated in a number of physio/pathological conditions. ${ }^{27}$ Metabolic maps are available that annotate the gene encoding for any given enzyme, the kinetic parameters for each substrate, the required cofactors, the allosteric regulators, but the simple measurement of enzyme expression and of metabolite levels is not sufficient to indicate which metabolic pathway is utilized in any given cell and at any given time. Metabolome analysis based on stable labeled precursors and a systems modeling approach are the tools that are being developed, to describe how cells regulate their global metabolism. ${ }^{8,28-30}$ Many maps of cancer metabolic rewiring (CMR) are reported in the literature, ${ }^{31-33}$ but they do not dedicate specific attention to the redox balance. The map we present in Figures 1 and 2 does so.

If we consider only the carbon flux, then Glutamine (Gln) is taken up by the cell through a transporter (Slc1a5), whose expression is stimulated by several oncogenic signaling pathways. ${ }^{11,34}$ Then Gln is deaminated to glutamate (Glu) by cytoplasmic GLS1 and transferred by Slc25a11 mitochondrial carrier into the mitochondrial matrix. Otherwise, Gln may be transported into mitochondria, where it may be deaminated to Glu by GLS2. It is interesting to recall that Myc controls GLS1 expression, ${ }^{14}$ while $p 53^{35,36}$ and p53-family members control GLS2. ${ }^{37-39}$ In turn, glutamate dehydrogenase (GDH) converts glutamate into a-ketoglutarate $(\mathrm{Akg})$. This reaction of reductive carboxylation is performed in mitochondria by isocitrate dehydrogense enzyme (IDH) 2, but may be performed also 


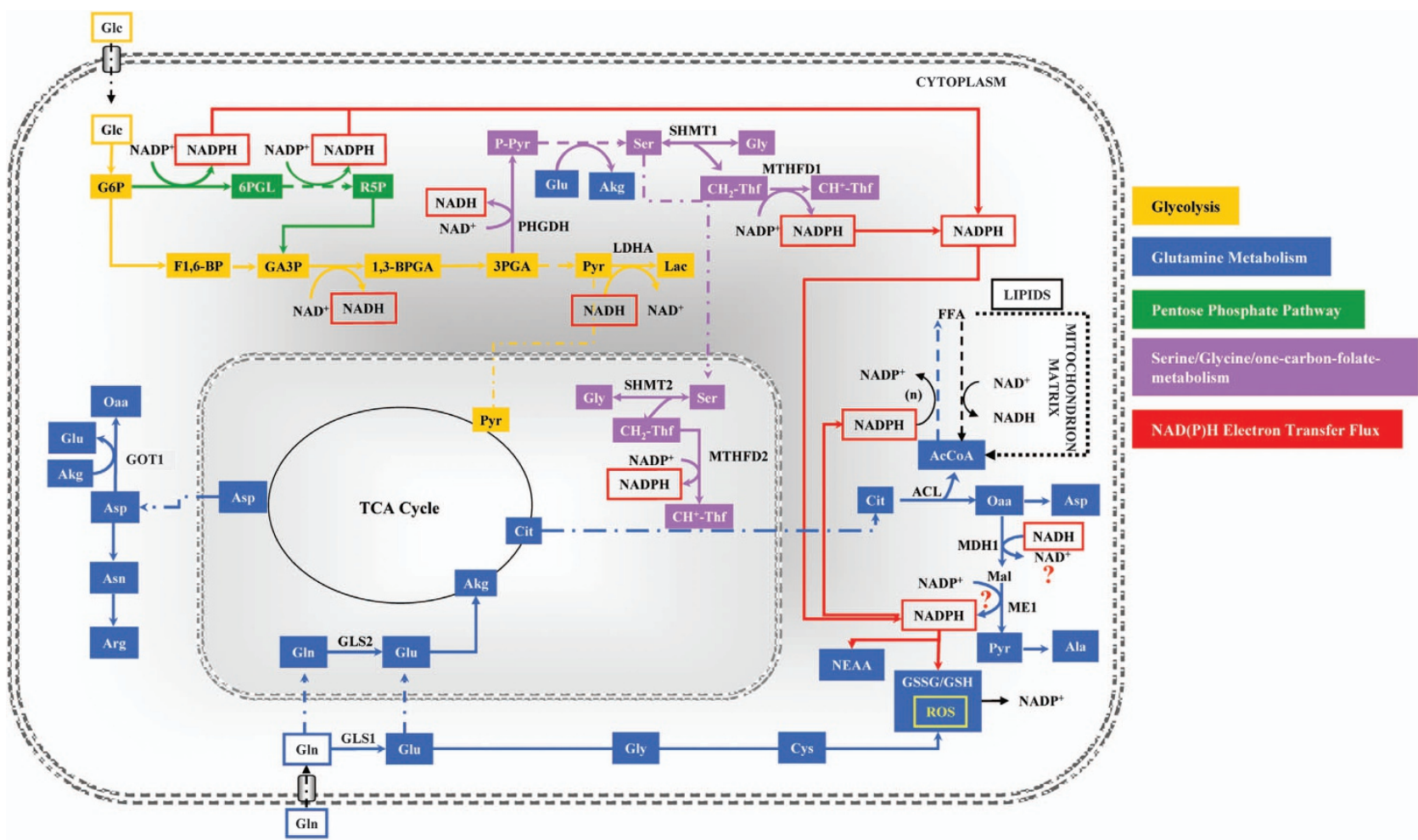

Figure 2 Concept map of the cancer metabolic rewiring. Large glycolytic flux and complex I dysfunction are required to sustain glutamine reductive carboxylation. Yellow arrows identify glycolysis. Blue arrows identify glutamine metabolism, green arrows identify pentose phosphate pathway (PPP), bright Lilac identifies serine/glycine/one-carbonfolate-metabolism, and red arrows identify an NAD(P)H electron transfer flux (ETF). NAD(P)H ETF originates from serine diversion pathway, sustains lipid synthesis, ROS quenching by GSH and reductive steps of non-essential amino-acid (NEAA) synthesis. Abbreviations: 1,3BPGA, bisphosphoglycerate; 3PGA, 3phosphoglycerate; 6PGL, 6-phosphogluconolactone; AcCoA, acetyl-CoA; ACL, ATP citrate lyase; Akg, $\alpha$-ketoglutarate; Ala, alanine; Arg, arginine; Asn, asparagine; Asp, aspartate; $\mathrm{CH}^{+}$-Thf, 5-methylenetetrahydrofolate; $\mathrm{CH}_{2}$-Thf, 5,10-methylenetetrahydrofolate; Cit, citrate; Cys, cysteine; F1,6BP, fructose 1,6 biphosphate; FFA, fatty acids; G6P, glucose 6-phosphate; GA3P, glyceraldehyde 3-phosphate; Glc, glucose; Gln, glutamine; Glu, glutamate; Gly, glycine; GOT, glutamic-oxaloacetic transaminase; GSH, glutathione reduced; GSSG, glutathione oxidized; Lac, lactate; LDHA, lactate dehydrogenase; MDH 1, malate dehydrogenase; ME, malic enzyme; MTHFD1 and 2, methylenetetrahydrofolate dehydrogenase (NADP ${ }^{+}$dependent); NEAA, non-essential amino acids; Oaa, oxalacetate; PPP, pentose phosphate pathway; P-Pyr, 3-phosphohydroxypyruvate; Pyr, pyruvate; R5P, ribose 5-phosphate; ROS, reactive species oxygen; Ser, serine; SHMT1 and 2, serine hydroxymethyltransferase

in the cytosol by IDH1 Two pathways have been described for the Akg utilization in cancer cells, one counterclockwise (pathway B) and the other clockwise (pathway A). In pathway $B$ (Figure 1), Akg is carboxylated to Isocitrate (IsoCit), which is converted into Citrate (Cit) and then exported into the cytoplasm. The cytosolic enzyme ATP-citrate-lyase (ACL) splits it to oxalacetate (Oaa) and Acetyl-CoA (AcCoA). AcCoA is then utilized as a building block for lipid biosynthesis. ${ }^{40,41}$ In pathway A, Akg follows normal TCA cycle steps until Oaa, which is then converted into aspartate (Asp) by aspartate transaminase (GOT2) and exported into the cytoplasm. In turn, Asp may be transformed into Asparagine (Asn) and Arginine (Arg) to be used for protein synthesis.

\section{Involvement of NADH and NADPH in CMR}

Many reactions of pathways $A$ and $B$ (Figure 1) are red/ox reactions, which are strongly affected by the availability of the two specific coenzymes $\operatorname{NAD}(\mathrm{H})$ and $\operatorname{NADP}(\mathrm{H})$. With regard to the reactions that take place in the mitochondrial matrix, as previously recalled, Complex I dysfunction is a fairly common event in cancer cells, ${ }^{42,43}$ but other mutations can occur in the TCA cycle enzymes, fumarate hydratase $(\mathrm{FH})$ and succinate dehydrogenase $(\mathrm{SDH}),{ }^{44-46}$ or in other complexes of the electron transfer chain (ETC), such as Complex III. ${ }^{47}$ All these alterations have been shown to promote glutamine utilization by reductive carboxylation. ${ }^{41,47,48}$ An in vitro analysis of this reaction indicates that, for adequate citrate production to occur, through isocitrate dehydrogenase (IDH)-catalyzed reductive carboxylation, a high mitochondrial NADPH/NADP ${ }^{+}$ ratio is required. ${ }^{49}$ From this standpoint, although pathway $B$ appears to be redox balanced, involving two reactions, the first one $(\mathrm{GDH})$ generating NADPH and the other one IDH which consumes it, it is not surprising that the enhanced utilization of glutamine requires, in vivo, the activity of nicotinamide nucleotide transhydrogenase (NNT), a mitochondrial enzyme that transfers electrons from NADH to NADPH. ${ }^{50}$ In addition, in cancer cells with defective $\mathrm{FH}$ or with Complex III dysfunction, the oxidation of Akg in the TCA cycle (path A of Figure 1) is required to sustain concomitantly reductive carboxylation to Isocitrate, thereby indicating an overflow of reducing equivalents to be used for conversion of Akg into Isocitrate. ${ }^{48}$ While succinate accumulates in $\mathrm{FH}$ defective cancer cells, it does not do so in cancer cells harboring a defective Complex I. ${ }^{48}$ In the latter cells, each molecule of Akg is converted into Oaa producing two molecules of $\mathrm{NADH}$ and one molecule of $\mathrm{FADH}_{2}$. As previously indicated, NADH may be converted into 
NADPH by NNT, powering the reductive carboxylation reaction, or it may feed the dysfunctional ETC to produce ATP and consume oxygen. The residual activity of the ETC in K-ras transformed fibroblasts is around $50 \%$ of normal cells due to decreased Complex I activity and different expression levels of several OXPHOS genes. ${ }^{10}$ Consistent with these data, Hu et $a^{\rho}$ have shown that in vitro activation of $\mathrm{K}$-ras ${ }^{\mathrm{G} 12 \mathrm{~V}}$ caused a disruption of Complex I. Specifically, using the SILAC (stable isotope labeling with amino acids in cell culture)-based mass spectrometry method, the authors have observed decreased levels of several Complex I components (NDUFA2, NDUFA4, NDUFA5, NDUFA11, NDUFA12, NDUFA13, NDUFB4, NDUFB6, and NDUFB7). ${ }^{9}$ To date, several interesting works have shown the link between oncogenic K-ras and mitochondrial dysfunction of cancer cells. ${ }^{51-54}$ Moreover, this connection probably causes the metabolic reprogramming toward glutamine utilization in the TCA cycle to maintain cellular redox homeostasis. ${ }^{8,54,55}$ In conclusion, the inactivation of components of the ETC or the TCA cycle (characteristic of most cancer cells) sets the conditions which are able to promote glutamine utilization by reductive carboxylation. In fact, it leads to an increase in $\mathrm{NADH} / \mathrm{NAD}^{+}$in the mitochondrial matrix, which, by action of NNT, determines an increase in NADPH/NADP ${ }^{+}$, required to sustain reductive carboxylation of $\mathrm{Akg}$.

Although many findings are available on the biological responses elicited by glutamine utilization in cancer cells, the fate of the products of glutamine metabolism, citrate and aspartate, that are exported from the mitochondria to the cytoplasm, is in part undetermined as yet. We present below a new interpretation that supports the notion that it is glycolysis, which sustains the reducing equivalents for a complete glutamine utilization.

In cancer cells, the expression of ACL is strongly upregulated $^{56}$ and reductive glutamine metabolism, catalyzed by IDH1 and IDH2, results in an enhanced lipid biosynthesis. ${ }^{41}$ Related to this, overexpression of fatty acid synthase confers growth and survival advantages on cancer cells, ${ }^{57}$ while, on the contrary, inhibition of ACL suppresses tumor cells growth. ${ }^{58}$ Taken together, these findings indicate that a sustained production of lipids from AcCoA derived from glutamine takes place in cancer cells and is necessary to support their growth and survival.

Which are the metabolic sources of the NADPH required to convert AcCoA into lipids? In actively proliferating cells, the oxidative pentose phosphate pathway (PPP) has long been considered to be the major source of NADPH, with malic enzyme also being important in some cell types. ${ }^{11,59}$ Recently, it has been reported that a comparable contribution comes from the Tetrahydrofolate (THF)-dependent pathway. ${ }^{60}$ This pathway stems from serine, whose production from the glycolytic intermediate 3-Phosphoglyceric acid (3Pga) requires phosphoglycerate dehydrogenase $(\mathrm{PHGDH})$, an enzyme that generates NADH and whose function is strongly stimulated in many cancer cells (Figure 2). ${ }^{61-64}$ In the conversion from serine into glycine (stimulated in cancer cells) methylenetetrahydrofolate is formed, whose oxidation generates NADPH (Figure 1). ${ }^{64}$ Quantitative flux analysis indicates that the synthesis of lipids is the pathway that utilizes a large share of NADPH production. ${ }^{60}$ By tracing hydrogen in compartmentalized pathways that utilize NADPH as a cofactor, fluxes of reactions that take place either within mitochondria or cytosol have been estimated. ${ }^{65}$ This interesting new metabolomic method, allowing cytosolic and mitochondrial NADPH compartment identification, has shown that the majority of mitochondrial NADPH and glycine is generated by mitochondrial serine hydroxymethyltransferase (SHMT2) and methylenetetrahydrofolate dehydrogenase 2 (MTHFD2). Consistent with Lewis and colleagues, a mathematical model of folate metabolism compartmentalization has identified in embryonic tissues and cancer cells, that SHMT2 and MTHFD2 mainly supported cytosolic purine and pyrimidine synthesis via the export of formate. ${ }^{66}$ Moreover, a very recent work has shown that SHMT2 was activated in cancer cells with oncogenic myc under hypoxic condition and knockdown of SHMT2 showed a reduced cellular NADPH/NADP ${ }^{+}$ratio. $^{67}$ Following this approach, it will be possible to investigate whether $\mathrm{NADH}$ generated by $\mathrm{PHGDH}$ is converted into NADPH by the combined activity of malate dehydrogenase $(\mathrm{MDH} 1)$ and malic enzyme (ME1) (Figure 2). If this is the case, then one may answer the question whether the reducing power generated by the serine/glycine glycolitic diversion is exploited to increase the NADPH/NADP ${ }^{+}$ratio, thereby stimulating non-essential amino-acid (NEAA) synthesis and increasing the reduced/oxidized glutathione ratio (GSH/ GSSG) required for ROS quenching (Figure 2).

It is well known that the large majority of cancer cells are unable to grow only on glutamine, but require active glucose metabolism via glycolysis ${ }^{23}$ and PPP. ${ }^{68}$ The described serine/glycine diversion of glycolysis may then be viewed as a means for promoting glutamine utilization that fuels growth and survival of cancer cells.

\section{Role of CMR for Growth and Survival}

To better understand the physiological role of CMR, it is interesting to define the supply of building blocks for growth that comes from each of its two processes: glycolysis and glutamine utilization by reductive carboxylation. Given that proteins are the largest cellular macromolecular components, about $70 \%$ of a dry weight of 500 pgr per cell, for standard mammalian cells, ${ }^{26}$ we utilize the reported fractional contribution of each amino acid in total protein and calculate the corresponding number of molecules for each NEAA that has to be obtained from metabolism (Figure 3a). The assignment to known synthetic pathways shows that at least $50 \%$ of the amino acids required for 'de novo' protein synthetic activity of cancer cells derives from glutamine metabolism (Figure $3 b$ ). Due to the lack of specific tracing experiments, the amino acids derived from pyruvate have been assigned to the glycolytic pathway, although pyruvate may also be produced from glutamine (Figure 1). Of course this is only a crude approximation of the role of glutamine utilization in cancer cell growth, since glutamine provides also a large aliquot of nucleotides and lipids.

Proteome-wide analysis of protein turnover rates in growing mammalian cells has shown that most proteins have half-lives ranging from minutes to a few hours. Hence, a large part of protein biosynthetic activity is devoted to maintaining steadystate levels and not to producing proteins for the new cells. ${ }^{69}$ In this context, the process of mitophagy (autophagic elimination of mitochondria), promoted by excess nutrient availability, and 

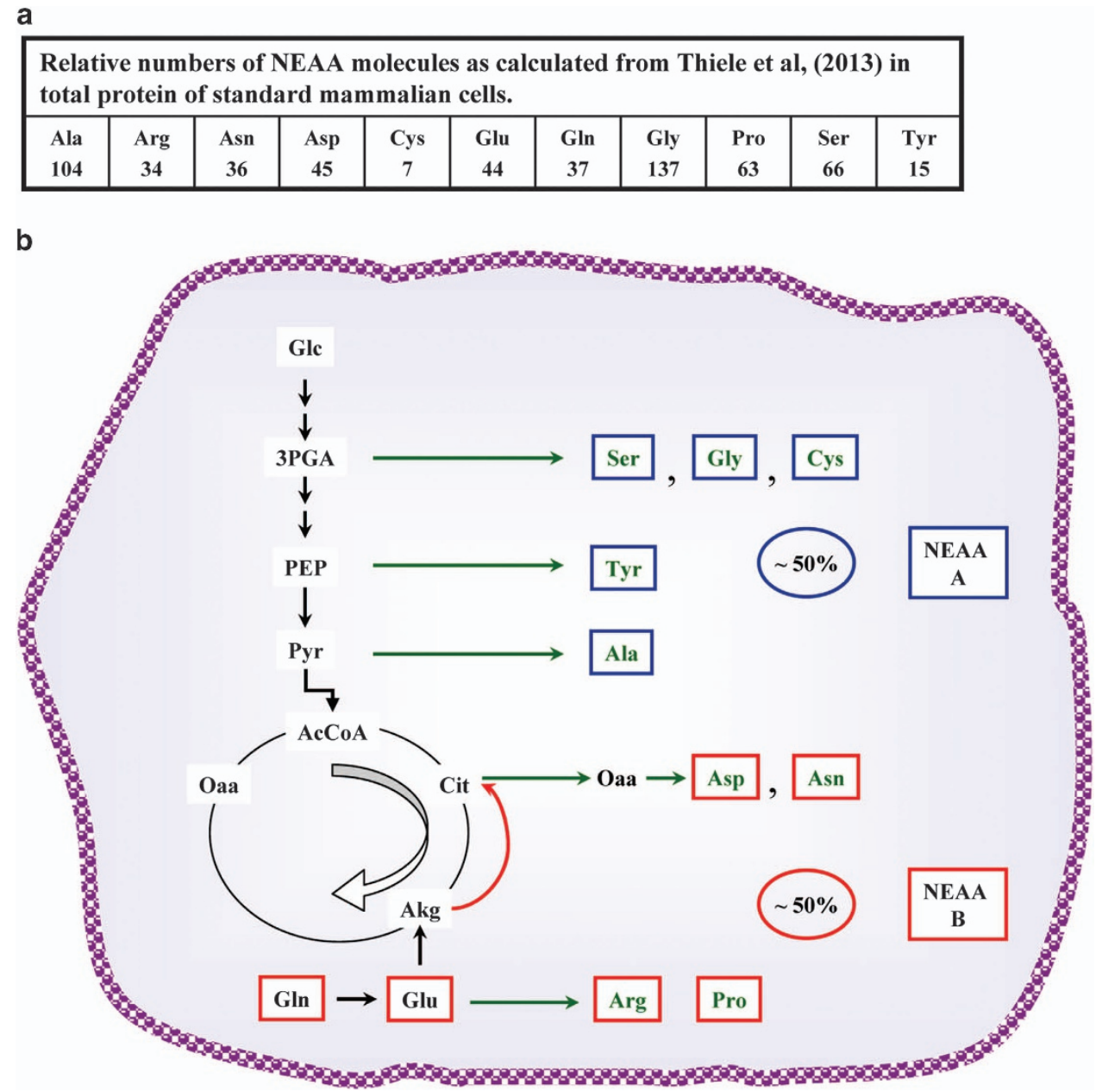

Figure 3 Schematic representation of NEAA synthesis. (a) Schematic fractional contribution of each amino acid in total protein and the corresponding number of molecules calculated for each NEAA that have to be obtained from metabolism. (b) Schematic representation of NEAA synthesis derived from glutamine utilization in cancer cells

resulting in mitochondrial dysfunction and depolarization, ${ }^{70}$ is of particular interest. Fragmentation of mitochondria, which precedes mitophagy, is observed in fact in cancer cells and takes place when glucose and glutamine uptake are stimulated. ${ }^{59}$ As for ATP utilization, the amount spent to sustain 'de novo' cell growth is much less than that required for basal cellular maintenance. ${ }^{61,62}$ The amino acids derived from extensive protein degradation may be recycled or enter the urea cycle producing polyamines and proline, both found to be upregulated in cancer cells. ${ }^{71,72}$

Considering that the amino acids derived from glutamine are the ones obtained from Oaa and Akg, which are also intermediates of the TCA cycle, what is the physiological advantage for cancer cells to synthesize amino acids using glutamine by reductive carboxylation as compared with normal cells that derive them by using skeleton carbon from glucose and amino groups from glutamine?

The first obvious difference is to have immediately available GIn and Glu from the environment, thereby sparing cells their biosynthesis starting from Akg, produced by the TCA cycle. The second one is that practically all Gln, no matter which metabolic route is followed, is converted to Oaa. Accordingly, NEAA (B) shown in Figure $3 b$ may be produced at a fast rate.
Moreover, the CMR ensures that the rate of glutamine utilization is coordinated with the stimulated rate of glycolysis, which characterizes cancer cells. Glycolysis in fact has a master control over the glutamine pathway, exerted by the regulation of the serine/THF diversion, with the rate of NADPH production (and possibly NADH) being exploited in several steps of the glutamine pathway. Normal cells instead control the rate of production of NEAA in a more rigid way: it is the rate of refilling of Oaa from pyruvate that allows the utilization of the intermediates Akg and Oaa away from the TCA cycle.

Taken together, the findings discussed so far point, for the first time, to a strong regulatory role of $\operatorname{NAD}(\mathrm{H})$ and $\operatorname{NADP}(\mathrm{H})$ metabolism in sustaining cancer cell growth.

\section{NADH and Tumor Aggressiveness}

The relevance of NAD metabolism in tumor development is supported by the results of a number of experiments, in which the availability of $\mathrm{NAD}(\mathrm{H})$ has been manipulated by genetic or biochemical means. Recently, considerable attention has been focused on NAMPT (Figure 4) as a potential therapeutic target, since several reports have shown its overexpression in several kinds of ${ }^{73,74}$ and, conversely, its downregulation by 


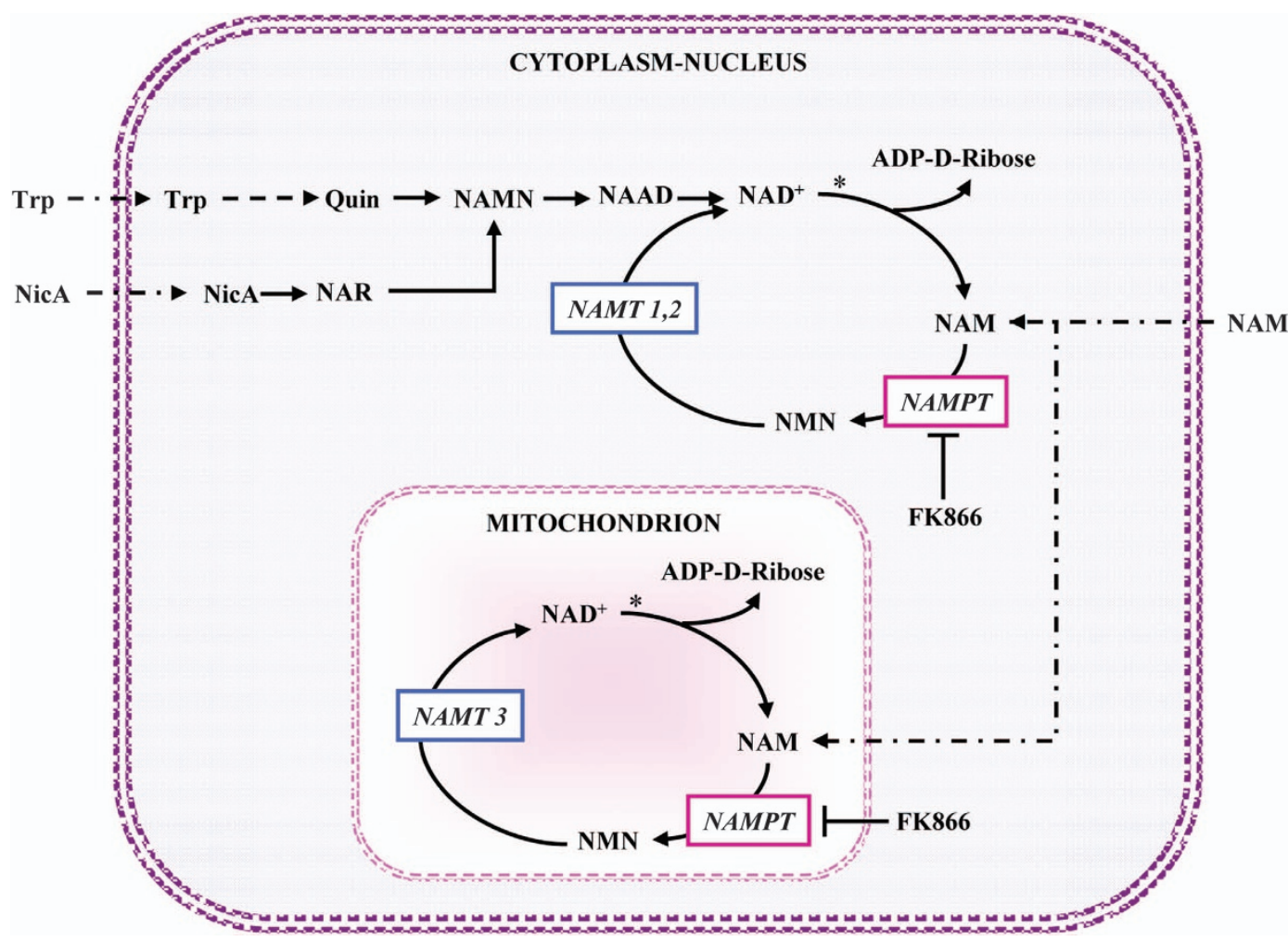

Figure 4 Map of the NAD \pm biosynthetic pathway. To clarify the interpretation of the 'in vivo' experiments described in the test, the NAD' biosynthetic pathway is schematically reported: $\mathrm{NAD}^{+}$can be synthesized from tryptophan (de novo synthesis) or nicotinic acid -NicA- or nicotinamide -Nam- (salvage pathway). NAD+ synthesis via tryptophan is performed by several reactions that lead to quinolinic acid (Quin) synthesis: this is next converted into nicotinic acid mononucleotide (NAMN) and NAMN to desamido-NAD (NAAD) and eventually into $N A D^{+}$. Diversely, in the major salvage pathway of NAD synthesis, NAM can be converted into nicotinamide mononucleotide (NMN) by nicotinamide phosphoribosyltransferase (NAMPT) and NMN is further converted into NAD by NMN adenylyltransferase (NMNAT 1 and 2 nuclear and cytoplasmic isoforms respectively, NMNAT3 mitochodrial isoform). The asterisk indicates a reaction, catalyzed by NAD ${ }^{+}$glycohydrolase, which bears close similarity with the family of ADP ribosyl cyclases. However, all the NAD'-transforming enzyme families mentioned above (i.e., Mono ADP-ribosyl transferases, $\mathrm{PARP}_{\mathrm{s}}$, sirtuins) share with ADP ribosyl cyclases/NAD glycohydrolases the property of releasing NAM from NAD

genetic or chemical means suppresses tumor cell growth in vitro and in tumor xenografts as well. ${ }^{75-77}$ One of the most widely used inhibitors of NAMPT is FK866, which is under development as an anticancer drug. ${ }^{24,78}$

When human breast cancer cells, defective in Complex I activity, are transfected to express yeast NADH dehydrogenase, Ndi1, their ability to oxidize mitochondrial NADH is restored and, at the same time, their tumor forming ability in xenografts is substantially reduced. ${ }^{79}$ Given that the Ndi1 expression enhances the $\mathrm{NAD}^{+} / \mathrm{NADH}$ ratio in whole cells and mitochondria, the effect was analyzed by supplying $\mathrm{NAD}^{+}$ precursors, NicA and $\mathrm{NAM}^{80}$ to the same Complex l-defective breast cancer cells. The tumor forming ability of xenografts is dramatically reduced by treatment with NicA and NAM, both able to refill the level of $\mathrm{NAD}^{+}$in mitochondrial and in cytoplasmic/nuclear compartments (Figure 4). Moreover, the supplying of $\mathrm{NAD}^{+}$precursors in drinking water, after primary breast cancer removal in animal models, increases survival. ${ }^{79}$ Consistent with the previous observations, these findings confirm that dysfunction of the ETC or of TCA cycle enzymes is required to trigger glutamine utilization by reductive carboxylation and suggest the possibility of a regulatory role of $\mathrm{NADH} / \mathrm{NAD}^{+}$ratio in the onset of glutamine utilization.

Treatment with FK866 strongly reduces the NAD ${ }^{+}$available to the glyceraldehyde 3-phosphate dehydrogenase (GAPDH). This inhibition, on the one hand, leads to an accumulation of glycolytic intermediates upstream of the inhibited GAPDH step, with an increased flux into the PPP ${ }^{68}$ and, on the other hand, reduces the carbon flow into the serine/THF pathway and, in a less stringent way and in the presence of non-limiting glutamine, in the TCA cycle as well. ${ }^{77}$

The dependence of cancer cell growth on $\operatorname{NAD}(\mathrm{H})$ seems to be far more complex than previously described. For instance, it has been shown that the decrease in $\mathrm{NAD}^{+}$levels, obtained by inhibiting with shRNA the expression of NAMPT, could make tumor cells more aggressive, ${ }^{79}$ while the treatment with FK866, that targets the same enzyme ${ }^{81}$ leads to cancer cell death both in vitro and in vivo. ${ }^{76}$ This discrepancy supports the notion that FK866, besides featuring an inhibitory effect on NAMPT, could have additional cytotoxic properties on cancer cells. $^{79}$

Moreover, recent data have demonstrated that pharmacological inhibition of NAMPT by FK866 can be counterbalanced by rescue of $\mathrm{NAD}^{+}$production from precursors other than NAM, notably NMN and Nicotinamide riboside (NR). ${ }^{82}$ Specifically, different FK866-treated tumor cells are affected by two ectoenzymes that are able to produce $\mathrm{NAD}^{+}$precursors. CD73 (generating NR from $\mathrm{NAD}^{+}$and NMN) enables, while CD38 (generating NAM that cannot be converted into $\mathrm{NAD}^{+}$because of NAMPT inhibition) impairs intracellular $\mathrm{NAD}^{+}$biosynthesis and consequently cell viability. 
Finally, to evaluate more critically the findings observed after FK866 treatment, it should be clarified whether the mitochondrial pool of $N A D(H)$ is sensitive to $F K 866$, given that literature data do not agree on this point. ${ }^{80,83}$

\section{Conclusions and Future Perspectives}

A lot of attention has been devoted, in recent years, to signaling and transcriptional events that promote cancer insurgence and progression, in an effort to develop a large number of new, target-oriented anticancer drugs. Unfortunately, the efficacy of these drugs in patients is often temporary, due to the frequent insurgence of molecular mechanisms of drug resistance. ${ }^{84}$ The recent awareness that many signaling pathways converge to a common bottom line, the metabolic rewiring, characterized by mitochondrial dysfunction, enhanced glycolysis and stimulated glutamine utilization through a reductive carboxylation reaction, offers the possibility of developing a new strategy for anticancer drug discovery. ${ }^{85}$ In fact, although a study of Marin-Valencia et al has shown that tumors, derived from transplantation of glioblastoma cells, used glucose to sustain anabolic processes accumulating glutamine, ${ }^{86}$ several works have demonstrated the efficacy of glutaminase inhibitors as BPTES (bis-2-(5-phenylacetamido-1,2,4-diathiazol-2-yl) ethyl sulfide) and CB-839 both in vitro and in vivo. ${ }^{21,87-91}$ Moreover, since the metabolic differences are mainly due to different kinds of tumor phenotype, a detailed metabolic profiling of human tumors should be performed to clarify the cancer types in which CMR takes place. For these cancer types, the concept map of the cancer rewired metabolism presented in this paper offers a rational basis for the development of a drug discovery strategy that aims to target rate-limiting nodes of the CMR. Moreover, this drug strategy to become really effective requires the evaluation of a number of quantitative aspects.

First of all, the utilization of various metabolomics techniques, such as gas chromatography-mass spectrometry (GC-MS) or liquid chromatography (LC-MS) combined with stable isotope tracers, in order to perform metabolic flux reconstruction and quantitative evaluation of enzyme function by metabolic flux analysis (MFA). ${ }^{8,29,41,92-94}$ In addition, this experimental/computational approach should permit the estimation of the fluxes in the various pathways (especially those less well known at the moment, such as the utilization of the reducing power produced by the serine/THF pathway ${ }^{60,65}$ in various cancer cells in vitro and possibly also in vivo). ${ }^{95}$ Given that present day technologies may accurately describe the metabolic profiles even in formalin-fixed paraffinembedded tumor biopsy samples, ${ }^{96}$ retrospective studies are feasible. More information on the role of mitochondrial and cytoplasmic $\mathrm{NAD}(\mathrm{P}) \mathrm{H} / \mathrm{NAD}(\mathrm{P})$ ratio in sustaining cancer metabolic rewiring may also be obtained by treatment with NAD pathway precursors or inhibitors, taking into careful consideration the aspects presently under debate. Development of quantitative dynamic models ${ }^{97}$ of the rewired cancer metabolism and of its regulation would add predictive ability to the new understanding (Box 1). ${ }^{85}$

Since the metabolic conditions required for tumorigenesis are Complex I dysfunction and enhanced glucose and glutamine utilization, the concept map presented herein may
Box 1. Computational models and design principles of cancer metabolic rewiring.

A deeper understanding of cancer metabolic rewiring requires the development of computational models ${ }^{85,97}$ that should consider at least all the metabolic reactions encompassed by the concept map of Figures 1 and 2.

A number of studies, generally dealing with specific pathways, such as glycolysis or TCA cycle, are reported in the literature. Two approaches are prevalent: quantitative mathematical models or genome-wide metabolic models, both of which present limitations as well as new insights. Of interest are, for instance, a genome-wide study of the Warburg effect that links metabolic steps to cancer cell migration ${ }^{101}$ and a mathematical modeling of the serine/glycine conversion rate in the NCI60 tumorderived cell lines that shows the contribution of glycine in purines, ATP, and NADPH production in cancer cells. ${ }^{102}$ The development of reliable, quantitative models linking metabolic rewiring to enhanced growth and survival of cancer cells is a next step. In the meantime, it may be of interest to comment on design principles that differentiate cancer cells from normal ones. It is often said that cancer cells have an inefficient metabolism since they only partially use the chemical potential of glucose, carrying on lactic fermentation and excreting lactate, ${ }^{103}$ while normal cells are more efficient since they fully utilize glucose in glycolysis and TCA cycle. But the situation is reversed for glutamine (the other relevant substrate for mammalian cells both 'in vitro' and 'in vivo'). Cancer cells use both carbon and nitrogen of glutamine, while normal cells use only nitrogen and excrete glutamic acid. ${ }^{8}$

In cancer cells, the production of reduced coenzymes for each glucose molecule entering either in the PPP or in serine/glycine pathway is different: for two molecules of NADPH formed in the first pathway, there are two of NADH and two of NADPH in the second one.

With regard to the relation of metabolism with growth, if one recalls that proteins are the largest component of biomass, it has to focus on the metabolic pathways bringing to amino acids (Figure 3 ). In normal cells, the rate of utilization of amino acids from TCA cycle is limited by the rate of refilling of Oaa to the TCA cycle from pyruvate and by availability of conditions that are going to affect the efficiency of TCA cycle as provider of amino acids (favorable ADP/ATP ratio, functionality and rate of execution of the electron transfer chain and availability of oxygen). In cancer cells, once the few enzymes of pathways $A$ and $B$ of Figures 1 and 2 are available, the rate of production of NEAA $B$ depends only upon the ability of PPP and/or of serine/glycine pathway to regenerate NADPH, while the rate of production of NEAA $A$ is expected to follow the enhanced rate of glycolysis.

In conclusion, in cancer cells glycolysis with its related pathways appears to have a master feed-forward control over glutamine utilization pathway, a condition that may be relevant in sustaining tumor forming ability 'in vivo'. 
also offer a new explanation for the reported increase of cancer incidence with aging. ${ }^{98}$ The reduction of Complex I activity characterizes the decline of metabolism during aging; ${ }^{99}$ this event originates a favorable ground for the oncogenic expression of somatic mutations able to stimulate nutrient uptake. Without the mitochondrial dysfunction related to aging (or to other causes), the simple stimulation of glucose and glutamine uptake might turn out to be non-tumorigenic. A deeper understanding of cancer metabolic rewiring proposed in Figures 1 and 2 and of its regulation may clarify whether sirtuins have a prevalent role in age-induced tumorigenesis, as proposed by $\mathrm{Wu}$ and colleagues, ${ }^{100}$ and may indicate ways to reactivate Complex I activity to prevent both aging and age-induced tumorigenesis.

\section{Conflict of Interest}

The authors declare no conflict of interest.

Acknowledgements. We wish to warmly thank Antonio De Flora for valuable comments and suggestions made on a previous version of this paper and the anonymous referees for thoughtful comments. This work has been supported by grants from the SysBioNet project, a MIUR initiative for the Italian Roadmap of European Strategy Forum on Research Infrastructures (ESFRI), to LA and from Tecnomed Foundation to DG.

1. Warburg O. On the origin of cancer cells. Science (New York, NY) 1956; 123: 309-314.

2. DeBerardinis RJ, Lum JJ, Hatzivassiliou G, Thompson CB. The biology of cancer: metabolic reprogramming fuels cell growth and proliferation. Cell Metab 2008; 7: 11-20.

3. Yuneva MO, Fan TW, Allen TD, Higashi RM, Ferraris DV, Tsukamoto T et al. The metabolic profile of tumors depends on both the responsible genetic lesion and tissue type. Cell Metab 2012; 15: 157-170.

4. Endo K, Oriuchi N, Higuchi T, lida $\mathrm{Y}$, Hanaoka $\mathrm{H}$, Miyakubo $M$ et al. PET and PET/CT using 18 F-FDG in the diagnosis and management of cancer patients. Int $\mathrm{J}$ Clin Oncol 2006; 11: 286-296.

5. Downward J. Targeting RAS signalling pathways in cancer therapy. Nat Rev 2003; 3 : $11-22$.

6. Dang CV. MYC on the path to cancer. Cell 2012; 149: 22-35.

7. Pylayeva-Gupta $Y$, Grabocka E, Bar-Sagi D. RAS oncogenes: weaving a tumorigenic web. Nat Rev 2011; 11: 761-774.

8. Gaglio D, Metallo CM, Gameiro PA, Hiller K, Danna LS, Balestrieri C et al. Oncogenic K-Ras decouples glucose and glutamine metabolism to support cancer cell growth. $\mathrm{Mol}$ Syst Biol 2011; 7: 523

9. Hu Y, Lu W, Chen G, Wang P, Chen Z, Zhou Y et al. K-ras(G12V) transformation leads to mitochondrial dysfunction and a metabolic switch from oxidative phosphorylation to glycolysis. Cell Res 2012; 22: 399-412.

10. Baracca A, Chiaradonna F, Sgarbi G, Solaini G, Alberghina L, Lenaz G. Mitochondrial Complex I decrease is responsible for bioenergetic dysfunction in K-ras transformed cells. Biochim Biophys Acta 2010; 1797: 314-323.

11. Son J, Lyssiotis CA, Ying H, Wang X, Hua S, Ligorio M et al. Glutamine supports pancreatic cancer growth through a KRAS-regulated metabolic pathway. Nature 2013; 496: 101-105.

12. Ying $\mathrm{H}$, Kimmelman AC, Lyssiotis $\mathrm{CA}$, Hua $\mathrm{S}$, Chu GC, Fletcher-Sananikone $\mathrm{E}$ et al. Oncogenic Kras maintains pancreatic tumors through regulation of anabolic glucose metabolism. Cell 2012; 149: 656-670.

13. Gaglio D, Soldati C, Vanoni M, Alberghina L, Chiaradonna F. Glutamine deprivation induces abortive s-phase rescued by deoxyribonucleotides in k-ras transformed fibroblasts. PLoS One 2009; 4: e4715.

14. Gao P, Tchernyshyov I, Chang TC, Lee YS, Kita K, Ochi T et al. c-Myc suppression of miR-23a/b enhances mitochondrial glutaminase expression and glutamine metabolism. Nature 2009; 458: 762-765.

15. Murphy TA, Dang CV, Young JD. Isotopically nonstationary $13 \mathrm{C}$ flux analysis of Myc-induced metabolic reprogramming in B-cells. Metab Eng 2013; 15: 206-217.

16. Wise DR, DeBerardinis RJ, Mancuso A, Sayed N, Zhang XY, Pfeiffer HK et al. Myc regulates a transcriptional program that stimulates mitochondrial glutaminolysis and leads to glutamine addiction. Proc Natil Acad Sci USA 2008; 105: 18782-18787.

17. Yuneva M, Zamboni $N$, Oefner $P$, Sachidanandam R, Lazebnik Y. Deficiency in glutamine but not glucose induces MYC-dependent apoptosis in human cells. J Cell Biol 2007; 178: 93-105.

18. Fendt SM, Bell EL, Keibler MA, Davidson SM, Wirth GJ, Fiske B et al. Metformin decreases glucose oxidation and increases the dependency of prostate cancer cells on reductive glutamine metabolism. Cancer Res 2013; 73: 4429-4438.
19. Le A, Cooper CR, Gouw AM, Dinavahi R, Maitra A, Deck LM et al. Inhibition of lactate dehydrogenase $A$ induces oxidative stress and inhibits tumor progression. Proc Natl Acad Sci USA 2010; 107: 2037-2042.

20. Xie H, Hanai J, Ren JG, Kats L, Burgess K, Bhargava $P$ et al. Targeting lactate dehydrogenase-a inhibits tumorigenesis and tumor progression in mouse models of lung cancer and impacts tumor-initiating cells. Cell Metab 2014; 19: 795-809.

21. Le A, Lane AN, Hamaker M, Bose S, Gouw A, Barbi J et al. Glucose-independent glutamine metabolism via TCA cycling for proliferation and survival in B cells. Cell Metab 2012; 15: 110-121.

22. Fan J, Kamphorst JJ, Mathew R, Chung MK, White E, Shlomi T et al. Glutamine-driven oxidative phosphorylation is a major ATP source in transformed mammalian cells in both normoxia and hypoxia. Mol Syst Biol 2013; 9: 712.

23. Ganapathy-Kanniappan S, Geschwind JF. Tumor glycolysis as a target for cancer therapy: progress and prospects. Mol Cancer 2013; 12: 152

24. Vander Heiden MG. Targeting cancer metabolism: a therapeutic window opens. Nat Rev 2011; 10: 671-684.

25. Hensley CT, Wasti AT, DeBerardinis RJ. Glutamine and cancer: cell biology, physiology, and clinical opportunities. J Clin Invest 2013; 123: 3678-3684.

26. Thiele I, Swainston N, Fleming RM, Hoppe A, Sahoo S, Aurich MK et al. A communitydriven global reconstruction of human metabolism. Nat Biotechnol 2013; 31: 419-425.

27. Loscalzo J, Kohane I, Barabasi AL. Human disease classification in the postgenomic era: a complex systems approach to human pathobiology. Mol Syst Biol 2007; 3: 124.

28. Metallo CM, Vander Heiden MG. Understanding metabolic regulation and its influence on cell physiology. Mol Cell 2013; 49: 388-398.

29. Hiller K, Metallo CM. Profiling metabolic networks to study cancer metabolism. Curr Opin Biotechnol 2013; 24: 60-68.

30. DeBerardinis RJ, Thompson CB. Cellular metabolism and disease: what do metabolic outliers teach us? Cell 2012; 148: 1132-1144.

31. Bryant KL, Mancias JD, Kimmelman AC, Der CJ. KRAS: feeding pancreatic cancer proliferation. Trends Biochem Sci 2014; 39: 91-100.

32. Dang CV. Links between metabolism and cancer. Genes Dev 2012; 26: 877-890.

33. Vander Heiden MG, Cantley LC, Thompson CB. Understanding the Warburg effect: the metabolic requirements of cell proliferation. Science (New York, NY) 2009; 324: 1029-1033.

34. Li B, Simon MC. Molecular Pathways: Targeting MYC-induced metabolic reprogramming and oncogenic stress in cancer. Clin Cancer Res 2013; 19: 5835-5841.

35. Hu W, Zhang C, Wu R, Sun Y, Levine A, Feng Z. Glutaminase 2, a novel p53 target gene regulating energy metabolism and antioxidant function. Proc Natl Acad Sci USA 2010; 107 7455-7460.

36. Suzuki S, Tanaka T, Poyurovsky MV, Nagano H, Mayama T, Ohkubo S et al. Phosphateactivated glutaminase (GLS2), a p53-inducible regulator of glutamine metabolism and reactive oxygen species. Proc Natl Acad Sci USA 2010; 107: 7461-7466.

37. Amelio I, Markert EK, Rufini A, Antonov AV, Sayan BS, Tucci $P$ et al. p73 regulates serine biosynthesis in cancer. Oncogene 2013; 33: 5039-5046.

38. Giacobbe A, Bongiorno-Borbone L, Bernassola F, Terrinoni A, Markert EK, Levine AJ et al. p63 regulates glutaminase 2 expression. Cell Cycle (Georgetown, Tex) 2013; 12 1395-1405.

39. Velletri T, Romeo F, Tucci P, Peschiaroli A, Annicchiarico-Petruzzelli M, Niklison-Chirou MV et al. GLS2 is transcriptionally regulated by p73 and contributes to neuronal differentiation Cell Cycle (Georgetown, Tex) 2013; 12: 3564-3573

40. Anastasiou D, Cantley LC. Breathless cancer cells get fat on glutamine. Cell Res 2012; 22 443-446

41. Metallo CM, Gameiro PA, Bell EL, Mattaini KR, Yang J, Hiller K et al. Reductive glutamine metabolism by IDH1 mediates lipogenesis under hypoxia. Nature 2011; 481: 380-384.

42. Birsoy K, Possemato R, Lorbeer FK, Bayraktar EC, Thiru P, Yucel B et al. Metabolic determinants of cancer cell sensitivity to glucose limitation and biguanides. Nature 2014; 508: 108-112.

43. Calabrese C, lommarini L, Kurelac I, Calvaruso MA, Capristo M, Lollini PL et a. Respiratory complex I is essential to induce a Warburg profile in mitochondria-defective tumor cells. Cancer Metab 2013; 1: 11

44. Tomlinson IP, Alam NA, Rowan AJ, Barclay E, Jaeger EE, Kelsell D et al. Germline mutations in $\mathrm{FH}$ predispose to dominantly inherited uterine fibroids, skin leiomyomata and papillary renal cell cancer. Nat Genet 2002; 30: 406-410.

45. Astuti D, Latif F, Dallol A, Dahia PL, Douglas F, George E et al. Gene mutations in the succinate dehydrogenase subunit SDHB cause susceptibility to familial pheochromocytoma and to familial paraganglioma. Am J Hum Genet 2001; 69: 49-54.

46. Killian JK, Kim SY, Miettinen M, Smith C, Merino M, Tsokos M et al. Succinate dehydrogenase mutation underlies global epigenomic divergence in gastrointestinal stromal tumor. Cancer Discov 3: 648-657.

47. Mullen AR, Wheaton WW, Jin ES, Chen PH, Sullivan LB, Cheng T et al. Reductive carboxylation supports growth in tumour cells with defective mitochondria. Nature 2011 481: 385-388.

48. Mullen AR, Hu Z, Shi X, Jiang L, Boroughs LK, Kovacs Z et al. Oxidation of alphaketoglutarate is required for reductive carboxylation in cancer cells with mitochondria defects. Cell Rep 2014; 7: 1679-1690.

49. Leonardi R, Subramanian C, Jackowski S, Rock CO. Cancer-associated isocitrate dehydrogenase mutations inactivate NADPH-dependent reductive carboxylation. $J$ Biol Chem 2012; 287: 14615-14620. 
50. Gameiro PA, Laviolette LA, Kelleher JK, lliopoulos O, Stephanopoulos G. Cofactor balance by nicotinamide nucleotide transhydrogenase (NNT) coordinates reductive carboxylation and glucose catabolism in the tricarboxylic acid (TCA) cycle. J Biol Chem 2013; 288 : 12967-12977.

51. Chun SY, Johnson C, Washburn JG, Cruz-Correa MR, Dang DT, Dang LH. Oncogenic KRAS modulates mitochondrial metabolism in human colon cancer cells by inducing HIF-1alpha and HIF-2alpha target genes. Mol Cancer 2010; 9: 293.

52. Neuzil J, Rohlena J, Dong LF. K-Ras and mitochondria: dangerous liaisons. Cell Res 2011; 22: $285-287$

53. Ralph SJ, Rodriguez-Enriquez S, Neuzil J, Saavedra E, Moreno-Sanchez R. The causes of cancer revisited: 'mitochondrial malignancy' and ROS-induced oncogenic transformation why mitochondria are targets for cancer therapy. Mol Aspects Med 2010; 31: 145-170.

54. Weinberg F, Hamanaka R, Wheaton WW, Weinberg S, Joseph J, Lopez M et al. Mitochondrial metabolism and ROS generation are essential for Kras-mediated tumorigenicity. Proc Natl Acad Sci USA 2010; 107: 8788-8793.

55. Blum R, Kloog Y. Metabolism addiction in pancreatic cancer. Cell Death Dis 2014; 5: e1065.

56. Zaidi N, Swinnen JV, Smans K. ATP-citrate lyase: a key player in cancer metabolism. Cancer Res 2012; 72: 3709-3714.

57. Menendez JA, Lupu R. Fatty acid synthase and the lipogenic phenotype in cancer pathogenesis. Nat Rev Cancer 2007; 7: 763-777.

58. Hatzivassiliou G, Zhao F, Bauer DE, Andreadis C, Shaw AN, Dhanak D et al. ATP citrate lyase inhibition can suppress tumor cell growth. Cancer Cell 2005; 8: 311-321.

59. Cairns RA, Harris I, McCracken S, Mak TW. Cancer cell metabolism. Cold Spring Harb Symp Quant Biol 2011; 76: 299-311

60. Fan J, Ye J, Kamphorst JJ, Shlomi T, Thompson CB, Rabinowitz JD. Quantitative flux analysis reveals folate-dependent NADPH production. Nature 2014; 510: 298-302.

61. Locasale JW, Cantley LC. Metabolic flux and the regulation of mammalian cell growth. Cell Metab 2011; 14: 443-451.

62. Locasale JW, Grassian AR, Melman T, Lyssiotis CA, Mattaini KR, Bass AJ et al. Phosphoglycerate dehydrogenase diverts glycolytic flux and contributes to oncogenesis. Nat Genet 2011; 43: 869-874.

63. Possemato R, Marks KM, Shaul YD, Pacold ME, Kim D, Birsoy K et al. Functional genomics reveal that the serine synthesis pathway is essential in breast cancer. Nature 2011; 476: 346-350.

64. Amelio I, Cutruzzola F, Antonov A, Agostini M, Melino G. Serine and glycine metabolism in cancer. Trends Biochem Sci 2014; 39: 191-198.

65. Lewis CA, Parker SJ, Fiske BP, McCloskey D, Gui DY, Green CR et al. Tracing compartmentalized NADPH metabolism in the cytosol and mitochondria of mammalian cells. Mol Cell 2014; 55: 253-263.

66. Nijhout HF, Reed MC, Lam SL, Shane B, Gregory 3rd JF, Ulrich CM. In silico experimentation with a model of hepatic mitochondrial folate metabolism. Theor Biol Med Model 2006; 3: 40

67. Ye J, Fan J, Venneti S, Wan YW, Pawel BR, Zhang J et al. Serine catabolism regulates mitochondrial redox control during hypoxia. Cancer Discov 2014; e-pub ahead of print 3 September 2014; doi:10.1158/2159-8290.CD-14-0250.

68. Patra KC, Hay N. The pentose phosphate pathway and cancer. Trends Biochem Sci 2014; 39: 347-354

69. Eden E, Geva-Zatorsky N, Issaeva I, Cohen A, Dekel E, Danon T et al. Proteome half-life dynamics in living human cells. Science (New York, NY) 2011; 331: 764-768.

70. Liesa M, Shirihai OS. Mitochondrial dynamics in the regulation of nutrient utilization and energy expenditure. Cell Metab 2013; 17: 491-506.

71. Gerner EW, Meyskens Jr FL. Polyamines and cancer: old molecules, new understanding. Nat Rev Cancer 2004; 4: 781-792.

72. Phang JM, Liu W. Proline metabolism and cancer. Front Biosci (Landmark edition) 2012 17: $1835-1845$.

73. Bowlby SC, Thomas MJ, D'Agostino Jr RB, Kridel SJ. Nicotinamide phosphoribosyl transferase (Nampt) is required for de novo lipogenesis in tumor cells. PLoS One 2012; 7 e40195.

74. Garten A, Petzold S, Korner A, Imai S, Kiess W. Nampt: linking NAD biology, metabolism and cancer. Trends Endocrinol Metab 2009; 20: 130-138.

75. Matheny CJ, Wei MC, Bassik MC, Donnelly AJ, Kampmann M, Iwasaki M et al. Nextgeneration NAMPT inhibitors identified by sequential high-throughput phenotypic chemical and functional genomic screens. Chem Biol 2013; 20: 1352-1363.

76. Chini CC, Guerrico AM, Nin V, Camacho-Pereira J, Escande C, Barbosa MT et al. Targeting of NAD metabolism in pancreatic cancer cells: potential novel therapy for pancreatic tumors. Clin Cancer Res 2013; 20: 120-130.

77. Tan B, Young DA, Lu ZH, Wang T, Meier TI, Shepard RL et al. Pharmacological inhibition of nicotinamide phosphoribosyltransferase (NAMPT), an enzyme essential for NAD+ biosynthesis, in human cancer cells: metabolic basis and potential clinical implications. J Biol Chem 2013; 288: 3500-3511.

78. Drevs J, Loser R, Rattel B, Esser N. Antiangiogenic potency of FK866/K22.175, a new inhibitor of intracellular NAD biosynthesis, in murine renal cell carcinoma. Anticancer Res 2003; 23: 4853-4858.

79. Santidrian AF, Matsuno-Yagi A, Ritland M, Seo BB, LeBoeuf SE, Gay LJ et al. Mitochondrial complex $\mathrm{I}$ activity and NAD+/NADH balance regulate breast cancer progression. J Clin Invest 2013; 123: 1068-1081.
80. Nikiforov A, Dolle C, Niere M, Ziegler M. Pathways and subcellular compartmentation of NAD biosynthesis in human cells: from entry of extracellular precursors to mitochondrial NAD generation. J Biol Chem 2011; 286: 21767-21778.

81. Hasmann M, Schemainda I. FK866, a highly specific noncompetitive inhibitor of nicotinamide phosphoribosyltransferase, represents a novel mechanism for induction of tumor cell apoptosis. Cancer Res 2003; 63: 7436-7442.

82. Grozio A, Sociali G, Sturla L, Caffa I, Soncini D, Salis A et al. CD73 protein as a source of extracellular precursors for sustained NAD+ biosynthesis in FK866-treated tumor cells. J Biol Chem 2013; 288: 25938-25949.

83. Pittelli M, Formentini L, Faraco G, Lapucci A, Rapizzi E, Cialdai F et al. Inhibition of nicotinamide phosphoribosyltransferase: cellular bioenergetics reveals a mitochondrial insensitive NAD pool. J Biol Chem 2010; 285: 34106-34114.

84. Wilson TR, Fridlyand J, Yan Y, Penuel E, Burton L, Chan E et al. Widespread potential for growth-factor-driven resistance to anticancer kinase inhibitors. Nature 2012; 487: 505-509.

85. Alberghina L, Gaglio D, Moresco RM, Gilardi MC, Messa C, Vanoni M. A systems biology road map for the discovery of drugs targeting cancer cell metabolism. Curr Pharm Des 2014; 20: 2648-2666.

86. Marin-Valencia I, Yang C, Mashimo T, Cho S, Baek H, Yang XL et al. Analysis of tumor metabolism reveals mitochondrial glucose oxidation in genetically diverse human glioblastomas in the mouse brain in vivo. Cell Metab 2012; 15: 827-837.

87. Galluzzi L, Kepp O, Vander Heiden MG, Kroemer G. Metabolic targets for cancer therapy. Nat Rev 2013; 12: 829-846.

88. Robinson MM, McBryant SJ, Tsukamoto T, Rojas C, Ferraris DV, Hamilton SK et al. Novel mechanism of inhibition of rat kidney-type glutaminase by bis-2-(5-phenylacetamido-1,2,4thiadiazol-2-yl)ethyl sulfide (BPTES). Biochem J 2007; 406: 407-414

89. Seltzer MJ, Bennett BD, Joshi AD, Gao P, Thomas AG, Ferraris DV et al. Inhibition of glutaminase preferentially slows growth of glioma cells with mutant IDH1. Cancer Res 2010; 70: 8981-8987.

90. Zhao Y, Butler EB, Tan M. Targeting cellular metabolism to improve cancer therapeutics. Cell Death Dis 2013; 4: e532.

91. Gross MI, Demo SD, Dennison JB, Chen L, Chernov-Rogan T, Goyal B et al. Antitumor activity of the glutaminase inhibitor CB-839 in triple-negative breast cancer. Mol Cancer Ther 2014; 13: 890-901.

92. Antoniewicz MR. 13C metabolic flux analysis: optimal design of isotopic labeling experiments. Curr Opin Biotechnol 2013; 24: 1116-1121.

93. Antoniewicz MR. Using multiple tracers for $13 \mathrm{C}$ metabolic flux analysis. Methods $\mathrm{Mol}$ Biol Clifton, NJ 2013; 985: 353-365.

94. Zhang J, Ahn WS, Gameiro PA, Keibler MA, Zhang Z, Stephanopoulos G. 13C isotopeassisted methods for quantifying glutamine metabolism in cancer cells. Methods Enzymol 2014; 542: 369-389.

95. Sugiura $Y$, Honda $K$, Kajimura $M$, Suematsu $M$. Visualization and quantification of cerebral metabolic fluxes of glucose in awake mice. Proteomics 2014; 14: 829-838.

96. Kelly AD, Breitkopf SB, Yuan M, Goldsmith J, Spentzos D, Asara JM. Metabolomic profiling from formalin-fixed, paraffin-embedded tumor tissue using targeted LC/MS/MS: application in sarcoma. PLoS One 2011; 6: e25357.

97. Shestov AA, Barker B, Gu Z, Locasale JW. Computational approaches for understanding energy metabolism. Wiley Interdiscip Rev 2013; 5: 733-750.

98. Frank SA. Age of cancer incidence. Dynamics of Cancer: Incidence, Inheritance, and Evolution. Princeton, NJ: Princeton University Press, 2007, pp 17-35.

99. Stefanatos R, Sanz A. Mitochondrial complex I: a central regulator of the aging process. Cell Cycle (Georgetown, Tex 2011; 10: 1528-1532.

100. Wu LE, Gomes AP, Sinclair DA. Geroncogenesis: metabolic changes during aging as a driver of tumorigenesis. Cancer Cell 2014; 25: 12-19.

101. Yizhak K, Le Devedec SE, Rogkoti VM, Baenke F, de Boer VC, Frezza C et al. A computational study of the Warburg effect identifies metabolic targets inhibiting cancer migration. Mol Syst Biol 2014; 10: 744

102. Tedeschi PM, Markert EK, Gounder M, Lin H, Dvorzhinski D, Dolfi SC et al. Contribution of serine, folate and glycine metabolism to the ATP, NADPH and purine requirements of cancer cells. Cell Death Dis 2013; 4: e877.

103. Doherty JR, Cleveland JL. Targeting lactate metabolism for cancer therapeutics. J Clin Invest 2013; 123: 3685-3692.

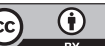

Cell Death and Disease is an open-access journal published by Nature Publishing Group. This work is licensed under a Creative Commons Attribution 4.0 International Licence. The images or other third party material in this article are included in the article's Creative Commons licence, unless indicated otherwise in the credit line; if the material is not included under the Creative Commons licence, users will need to obtain permission from the licence holder to reproduce the material. To view a copy of this licence, visit http://creativecommons.org/licenses/by/4.0 